quio principal contiene cartílago siendo más rígida que la de la arteria pulmonar. Además, la arteria pulmonar es un sistema de baja presión, con lo cuál cualquier compresión o distorsión aiterial puede ocasionar una redistribución marcada en el flujo sanjguíneo arteial; b) La hipoxia secundaria a la obstrucción del bronquio principal origina una vasoconstricción refleja arterial resultando en una hipoperfisión o ausencia de la captación del radiotrazador homolateral a la lesión. Es decir, el patrón gammagráfico de $\mathrm{P} / \mathrm{V}$ en el carcinoma broncogénico de localización hiliar, se caracteiza por defectos de actividad en el estudio de P/V en la mayoría de ocasiones no congnentes en su totalidad y con una mayor preservación de la ventilación pulmonar, pudiendo ocasionar un diagnóstico falso positivo de tromboembolismo pulmonar (3).

Es interesante, que tras efectuar un tratamiento quirurgico o radioterápico del carcinoma broncogénico intraluminal la alteración congruente de $\mathrm{Pi} /$, puede transformarse en una anomalía no congruente de $\mathrm{P} / \mathrm{V}$, ésto es, se puede corregir la hipoventilación secundaria a la lesión, pero resulta más diflcil normalizar los defectos de perfilsión. Este fenómeno representa la persistencia de una vasoconstriccióíiihipetensión vascular pulmonar mediada por el incremento de poliaminas en el pulmón secundario a la hipoxia (1).

De lo mencionado hasta ahora cabe resaltar dos hechos, que este patrón gammagráfico puede originar una estimación irreal del VEMS postoperatorio y que también puede ocasionar un diagnóstico inicial falso positivo de tromboembolismo pulmonar.

En definitiva, un diagnóstico diferencial exhaustivo en los pacientes que muestran una disminución global de la perfilsión pulmonar, resulta necesario para facilitar el manejo y evitar la yatrogenia derivada de un tratamiento inadecuado.

G. Ruíz Fernández, F. Carrión Valero*, C. Ferrer Albiach**, J.V. Balaguer Martínez, A. Mateo Navarro***, J. Marín Pardo**

Servicios de Urgencias, *Neumología, **Radioterapia. Hospital Clínico Universitario.***Departamento de Medicina. Universi dad de Valencia.

1. White I Jr, Jaulles AE Jr, Wagner HN Jr. The significance of unilateral absence of pulmollary artery perfusion by lung scalming. Am J Roentgenol Radium Ther Nucl Med 1971: 501-9.

2. Sutter CW, Stadalnik RC. Unilateral absence or near absence of pulmonary- perfusion on lung scaiuling. Semin Nucl Med 1995; 25: 72-4

3. Shill WJ, Jolulston EH. Jollston HW. Reversed abnormal -entilationperfusion scintigrap in endobronchial squamous cell carcinoma. Eur $\mathbf{J}$ Nucl Med 1982: 7: 523-5.

4. Shiao RT, Kostenbauder HB, Olson JW, Gillespie IN. Mechanisms of lung polyamine accumulation in chronic hipoxic pulmonary hypertension.Am J Physiol 1990; 259: 351-8.

\section{Fiebre prolongada y schwannoma maligno}

\section{Sr. Director:}

En un interesante artículo, Creagh et al (1) describen un caso de schwannoma maligno, neoplasia que puede manifestarse clíni camente de modos muy variados (2). Recientemente hemos tenido ocasión de atender a otra paciente con este raro tipo de tumor (3), que se presentó con fiebre prolongada y anemia.
Mujer de 14 años sin antecedentes de interés. Ingresa por tener desde hace dos meses fiebre alta persistente y astenia. A la exploración se observa un estado general conservado, temperatura axilar $39^{\circ} \mathrm{C}$ y palidez de piel y mucosas, mientras que todo lo demás es normal. Los análisis muestran: hemoglobina $8,5 \mathrm{~g} / \mathrm{dL}$, volumen corpuscular medio $70 \mathrm{fl}$, plaquetas $681.000 / \mathrm{mm}^{3}$, velocidad de sedimentación globular $120 \mathrm{~mm}$ en la primera hora, colesterol $134 \mathrm{mg} / \mathrm{dL}$, hierro $11 \mathrm{mg} / \mathrm{dl}$, fibrinógeno $587 \mathrm{mg} / \mathrm{dL}$, albúmina $2,8 \mathrm{~g} / \mathrm{dL}$ e IgE $394 \mathrm{U} / \mathrm{mL}$, mientras que son normales el resto de parámetros del hemograma, bioquímica, coagulación, proteinograma, anticuerpos antitisulares, hormonas tiroides, enzima conversora de la angiotensina, marcadores tumorales y análisis básico de orina. La ASLO es positiva, mientras que son negativos el resto de estudios microbiológicos, incluyendo cultivos de sangre, esputos, orina, heces y médula ósea, y serología de salmonelosis, brucelosis, enfermedad de Lyme, leishmaniasis, neumonías atípicas, mononucleosis infecciosa y hepatitis A, B y C. En la ecografía y el TAC abdominales se observa una tumoración de $5 \mathrm{~cm}$ de diámetro que parece depender del anexo izquierdo. Se propone la exéresis de dicha tumoración, pero se desestima reiteradamente, por considerarse que no es la causante de la fiebre. Por ello, se realizan las siguientes exploraciones complementarias: electrocardiograma, radiografías de tórax, TAC de la cabeza, oído izquierdo y tórax, tránsito intestinal completo, colonoscopia y ecocardiografía, todas las cuales son normales. Finalmente se realiza una laparotomía y se extirpa la tumoración y los ganglios linfáticos adyacentes, cuyo aspecto macroscópico es dudoso. El estudio anatomopatológico demuestra que el tumor es un schwannoma maligno y que los ganglios linfáticos se hallan invadidos por el tumor. Seguidamente la paciente queda afebril y asintomática.

Al schwannoma maligno también se le conoce con los nombres de neurofibrosarcoma, sarcoma neurogénico, y tumor maligno de la vaina de los nervios periféricos. Se trata de un tipo de cáncer generalmente agresivo. Tiene cierta asociación con la neurofibromatosis de von Recklinghausen, pero es muy poco frecuente en la población general (1). Nuestro caso posee grandes similitudes con el descrito por Creagh et al: ambos tumores aparecen en pacientes sin neurofibromatosis, y clínicamente se manifiestan con anemia y fiebre prologada. Especialmente llamativa resulta la presentación de los dos casos con síndrome febril de larga evolución, fenómeno bien conocido en diversas neoplasias (4), pero que según la literatura parece excepcional en el schwannoma maligno (5).

\section{B. Roca}

Unidad de Enfermedades Infecciosas. Hospital General de Castellón

1. Creagh R, Casado M, Elizande JM, Morales JA, Pujol E. Schwannoma maligno mediastínico en paciente sin enfermedad de Von Recklinghausen. An Med Interna (Madrid) 2001; 18: 531-3.

2. Cox JM, Alter M. Schwannoma: Challenging diagnosis. J Manipulative Physiol Ther 2001; 24: 526-8.

3. Ducatman BS, Scheithauer BW, Piepgras DG, Reiman HM, Ilstrup DM. Malignant peripheral nerve sheath tumor. A clinicopathologic study of 120 cases. Cancer 1986; 57: 2006-21.

4. Meis JM, Enzinger FM, Martz KL, Neal JA. Malignant peripheral nerve sheath tumors (malignant swannomas) in children. Am J Surg Pathol 1992; 16: 694-707.

5. Kleef R, Jonas WB, Knogler W, Stenzinger W. Fever, cancer incidence and spontaneous remissions. Neuroimmunomodulation 2001; 9: 55-64. 